\title{
Re-Emergence of Tetanus in a District Referral Hospital
}

\author{
Ariff $\mathrm{HH}$, Verghese A, Brian CMK \\ Department of Medicine, Hospital Teluk Intan \\ Jalan Changkat Jong, 36000 Teluk Intan, Perak
}

\begin{abstract}
Tetanus is an acute, potentially fatal disease caused by Clostridium tetani. It is characterized by generalized, painful spasms and rigidity of skeletal muscles. We present two fatal cases and discuss the potential causes that lead to mortality in cases of tetanus infection.
\end{abstract}

Keywords: Tetanus, creatine kinase in tetanus

\section{INTRODUCTION}

Tetanus is an acute, potentially fatal disease caused by Clostridium tetani. It is characterized by generalized, painful spasms and rigidity of skeletal muscles. Clostridium tetani can be found in the soil and feces of humans and animals. The spores enter the body through breaks in the skin e.g. puncture wounds or crush injuries. Clostridium tetani produces a potent neurotoxin (tetanospasmin), which interferes with inhibitory synapses in the skeletal muscles and spinal cord. This results in uncontrollable muscle contractions and classical clinical features like trismus, risus sardonicus and opisthotonus. In Malaysia there were 186 reported cases of tetanus between the years 2010 and 2013. Out of these, 23 died from the disease. ${ }^{1}$

\section{CASE 1}

A 52-year-old odd unemployed man presented to a district hospital with painful abdominal muscle spasm for four days. He did not complain of fever, vomiting or headache. The spasm and pain worsened progressively and he was referred for suspected peritonitis and intestinal obstruction.

Upon review, he was noted to be in respiratory distress and febrile. He had opisthotonus and the spatula test was positive. His abdominal muscles were tense and painful. Serum creatine kinase was noted to be markedly elevated at 19,288 IU/l. No obvious puncture wounds were seen. $\mathrm{He}$ was intubated and mechanically ventilated. Intramuscular (IM) ATT (anti-tetanus toxoid) and human

Corresponding author:

Dr Alice Verghese

Department of Medicine,

Hospital Teluk Intan

Jalan Changkat Jong,

36000 Teluk Intan, Perak

Email: alice_ver@yahoo.com

Tel: 0124326534 tetanus immunoglobulin were given. He was started on intravenous (IV) Ceftriaxone and IV Metronidazole. Morphine, midazolam and magnesium sulphate infusion were commenced to control the spasms. His creatine kinase level reduced to $5870 \mathrm{IU} / \mathrm{l}$. Tracheostomy was done for him in anticipation of prolonged ventilation. The patient remained febrile in the intensive care unit and continued to deteriorate. This was most likely due to a hospital-acquired pneumonia, as evidenced by consolidation on the chest radiograph. He developed acute kidney injury and coagulopathy, most likely due to sepsis. He succumbed to his illness and passed away ten days after his initial presentation. His endotracheal, blood and urine culture specimens were repeatedly negative.

\section{CASE 2}

This patient presented within a month of the first case. Both were not linked epidemiologically. A 20year-old Burmese fisherman presented with fever associated with painful muscle spasms of the jaw, neck and abdomen for three days. He had trismus but no opisthotonus. There were no obvious open wound or puncture site. He did not give any history of injury. His creatine kinase (CK) was elevated at $1996 \mathrm{IU} / \mathrm{L}$. He was given oral Baclofen, oral Clonazepam and intramuscular Haloperidol initially for spasm control.

Intramuscular ATT and Human Tetanus Immunoglobulin were given and he was started on IV Ceftriaxone and Metronidazole. He became increasingly restless with worsening of muscle spasms. He was intubated and started on Diazepam and magnesium sulphate infusion. His creatine kinase levels increased to a maximum of $2701 \mathrm{IU} / \mathrm{L}$ before declining progressively to $313 \mathrm{IU} / \mathrm{L}$. He developed a hospital-acquired pneumonia and required prolonged ventilation. This was supported by pneumonic changes on his chest radiograph. Cultures from the blood, urine and endotracheal secretions taken throughout admission did not isolate any organisms. On the 16th day of admission, 
he became restless and agitated before developing cardiorespiratory arrest. Oxygen saturation was maintained at $100 \%$ prior to collapse and there was no tachycardia or hypotension. He passed away despite active resuscitation.

\section{DISCUSSION}

Approximately one million cases of tetanus occur worldwide each year, with 300,000 to 500,000 deaths. ${ }^{2}$ The majority of cases occur in developing countries. In countries where there is an established tetanus immunization program, cases are mostly reported among the elderly, the indigenous 'orang asli' and migrant workers. Tetanus is not common in Malaysia and many doctors have not seen a case of tetanus. This can lead to a delay in diagnosis like our first case where the initial diagnosis was acute abdomen. Although there are general guidelines, many lack the experience and expertise in managing tetanus.

Patients usually require prolonged ventilation and tracheostomy. IM immunoglobulin has to be given as soon as possible as it binds only to the free toxins. As in both cases, the portal of entry may not be obvious. If present, a contaminated wound must be thoroughly debrided and cleaned. Patients must be given IV metronidazole to eradicate any remaining organism. Penicillin is now less preferred as it is believed to worsen spasm. Many early studies established the role of diazepam as a useful agent in tetanus ${ }^{3}$ and some of these compared the role of diazepam against other sedatives available at that time, such as chlorpromazine and phenobarbitone. Diazepam, a benzodiazepine derivative, is an effective tranquilizing and sedative agent with potent muscle relaxant and anti-convulsive properties. It binds to receptors in the spinal cord, brain stem, cerebellum, limbic system and cerebral cortex.

The binding of diazepam to the benzodiazepine receptor potentiates the inhibitory action of GABA upon the chloride channel, thereby enhancing GABA facilitated, inhibitory synaptic transmission. In both cases, creatine kinase appeared to be an indicator of prognosis and severity. High levels seemed to predict a worse outcome, while increasing levels denote uncontrolled disease activity.

Mortality is high with common causes of death being nosocomial infection. Secondary infections may include sepsis from indwelling catheters, hospital-acquired pneumonias, and decubitus ulcers. Aspiration pneumonia is a common late complication of tetanus, found in $50 \%-70 \%$ of autopsied cases. In the first case, the patient developed a hospitalacquired pneumonia which was evidenced by new area of consolidation the chest radiograph and newonset of fever while in ICU. Nosocomial infections are common because of the prolonged course of tetanus and remain an important cause of mortality. ${ }^{4}$ The cause of death in tetanus is rarely due to respiratory muscle paralysis. A group of patients in Sao Paulo, Brazil with generalized tetanus had been studied by Kurt Kloetzel in 1964 with the view of determining whether respiratory function was sufficiently impaired to be considered life-threatening.

It strongly suggested that respiratory failure is rarely a direct cause of death in tetanus. ${ }^{5}$ In the second case, the cause of death appeared to be cardiac, as sudden cardiac death is also one of the known complications of tetanus. He did not go into respiratory failure prior to collapse. There have not been many studies of the effects of tetanus on the cardiovascular system. Altered myocardial function may be because of persistently raised catecholamine levels ${ }^{6}$ but abnormal function may occur even in the absence of sepsis or high catecholamine levels. ${ }^{7}$

The factors said to be associated with poor prognosis in tetanus are unimmunized status, poor wound management; spasms at presentation, and delay in diagnosis and treatment. ${ }^{8}$ In both these patients the immunization status were unknown.

\section{CONCLUSION}

Although uncommon, tetanus remains a threat to at risk populations. A high index of suspicion is required to avoid missing a diagnosis of tetanus. Treatment is supportive and usually requires prolonged ventilation. High creatine kinase appears to be an indicator of poor prognosis and disease severity.

NMRR ID: NMRR-15-1171-26836

\section{Acknowledgements}

Special thanks to Puan Suhaya Binti Komari, Deputy Director of Pusat Informatik Kesihatan, Ministry of Health Malaysia for providing us with information on the latest tetanus statistics in Malaysia.

\section{REFERENCES}

1. Pusat Informatik Kesihatan, Ministry of Health Malaysia

2. Afshar M, Raju M, Ansell D, Bleck TP. Narrative review: tetanus-a health threat after natural disasters in developing countries. Ann Intern Med 2011; 154:329.

3. Joseph A, Pulimood BM: Use of diazepam in tetanus - a comparative study. Indian J Med Res 1978; 68: 489-491.

4. Andrew Michael Taylor, FRCA, Contin Educ Anaesth Crit Care Pain 2006; 6 (3): 101-104.doi: 10.1093/bjaceaccp/mkl01

5. Kurt Kloetzel, Studies on the Cause of Death in Tetanus : Human Tetanus as a Respiratory 
Problem. Chest 1964; 45(1): 63-71

6. Rose AG. Catecholamine-induced myocardial damage associated with phaeochromocytoma and tetanus. S Afr Med J 1974; 48: 1285-9

7. Iga K, Hori K. Rapidly progressive deteriorated left ventricular wall motion associated with tetanus. Jap J Med 1990; 29: 305-8

8. American Academy of Pediatrics. Red Book: 2009 Report of the Committee on Infectious Diseases. 28th ed. Elk Grove Village, IL: American Academy of Pediatrics; 2009. 
